\title{
Simulating NJL-Mechanism with Realistic Vector-Type \\ Confining Lagrangian
}

\author{
A.N. Mitra \\ Institute for Nuclear Theory, University of Washington \\ HN-12, Seattle, Washington 98195, USA \\ and \\ Department of Physics, University of Delhi \\ Delhi-110007, India

\section{Bani Miitra} \\ Department of Physics, University of Delhi \\ Delhi-110007, India
}

DOE/ER/40561--23

DE92 004376

\section{PREPARED FOR THE U.S. DEPARTMENT OF ENERGY UNDER GRANT DE-FG06-90ER40561}

This report was prepared as an account of work sponsored by the United States Government. Neither the United States nor any agency thereof, nor any of their employees, makes any warranty, express or implied, or assumes any legal liability or responsibility for the accuracy, completeness, or usefulness of any information, apparatus, product, or process disclosed, or represents that its use would not infringe privately owned rights. Reference herein to any specific commercial product, process, or service by trade name, mark, manufacturer, or otherwise, does not necessarily constitute or imply its endorsement, recommendation, or favoring by the United States Government or any agency thereof. The views and opinions of authors expressed herein do not necessarily state or reflect those of the United States Government or any agency therenf. 


\section{A.N.Mitra*, ** and Bani Mitra **}

*Institute for Nuclear Theory, HN-12, Univ of Washington, Seattle, Wh-98195 **Department of Physics,University of Delhi, Delhi-l10007, INDIA

\section{Abstract}

The concrete possioilities for a realistic simulation of the Nambu-Jona Lasino mechanism of dynamical mass generation are examined from the view-point of a Vector-type confining form of 4-fermion Lagrangian with a natural mass scale which substitutes for the canonical NJL-model of contact interaction, and is designed to keep a conscious link with spectroscopy. Such Lagrangians are known to lead (through the minimality condition on the effective Action) to both the Schwinger-Dyson and Bethe-Salpeter equations for the quark-mass operator $\Sigma(p)$ and the hadron-quark vertex function $\Gamma_{H}(\mathrm{q}, \mathrm{P})$ respectively. With a cholce of the interaction which corresponds to a Lorentz-covariant generalization of the familiar instantaneous approximation, the related Bethe-Salpeter equation already provides a good description of both $q \bar{q}$ and $q q q$ spectra, as well a Lorentz covariant access to different types of transition amplitudes via quark loops, within a common dynamical framework. With this pre-calabration, the model is subjected to the interlinked SDE and BSE for testing some key aspects of the NJL-self consistency mechanism by evaluating some basic constants. The results are: $\mathrm{m}_{\mathrm{NJL}}=300 \mathrm{Mev}, \mathrm{M}_{\pi}^{2} \sim \mathrm{m}_{c} ; M_{\sigma}=857 \mathrm{Mev} ;\langle q \bar{q}\rangle_{0}=(219 \mathrm{mev})^{3}$. From these values two independent estimates of $m_{c}$ are found as 21.8 and 17.8 (Mev). 
Introduction and motivation

Though the NJL-model is 30 years old ${ }^{1)}$ there has been a fresh spurt of activity on this model in recent times, with the originator himself taking a fresh look at its vast possibilities. ${ }^{2)}$ Its built-in mechanism for mass-generation

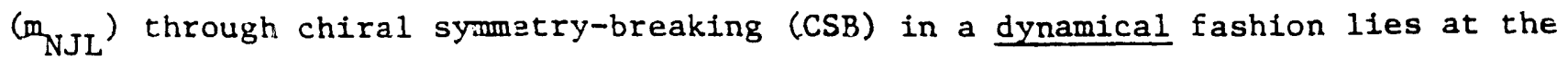
root of its natural appeal, especially in the context of light quarks (ud) whose smail current masses $\left(m_{c}\right)$ bring them closest to the chiral limit. This provides a formal rationale for the use of constituentusses $\left(m_{q}\right)$ via the politzer relation ${ }^{3}$ ) $m_{q}=m_{c}+m_{N J L}$ in most non-perturbative calculations, e.g., hadron spectroscopy in potential models, etc. Unfortunately the assumption of a contact 4-fermion coupling necessitating a cut-off $(\Lambda)$ for a fine-tuned determination of the dynamical mass $\left(\mathrm{m}_{\mathrm{NJL}}\right.$ ), severely restricts the predictive powers of the model. It is therefore of considerable interest to examine the possibilities of giving a wider meaning to the spirit of the NJL model without necessarily restricting the formalism to the literal features of a contact interaction.

It would indeed be an over simplification to suppose that the $N J$ model with a contact interaction per se (even with several chirally invariant mixtures of $\mathrm{S}, \mathrm{P}, \mathrm{V}, \mathrm{A}, \mathrm{T}$ couplings $\mathrm{S}^{4}$ and/or flavour extensions) could seriously address the dynamical issue of confinement, let alone provide a quantitative simulation of QCD. On the other hand, the basic spirit of the model- which will henceforth be termed the NJL-mechanism-- viz., mass generation through CSB as a result of a non-trivial solution of the Schwinger- Dyson Equation (SDE) for the mass operator $\Sigma(p)$, offers enough scope for more realistic constructions which imitate the confining features of QCD, while retaining the crucial chiral invariance property of the Lagrangian for almost massless quarks(ud). Indeed a simple and effective way to fulfil this condition would be to invoke a vector-exchange-type interaction Lagrangian(which respects global chiral symetry) with an extended structure designed to incorporate confinement by hand, in the spirit of traditional potential models 5 ) but suitably adapted to the requirement of Lorentz-invarience 6 ).

The construction proceeds logically as follows. Starting from a vector-type interaction Lagrangian with massless quarks, one constructs an effective action $W_{\text {eff }}$ in terms of bilocal fields in a more or less standard fashion ${ }^{7-10)}$. The quantity $W_{\text {eff }}$, when expanded around its stationary value w.r.t. the bilocal flelds, yields both the SDE for the quark mass function $\Sigma(p)$ and the BSE for the meson quark vertex function $\Gamma_{H}(q, P)$, the latter in the linearised approximation ${ }^{8)}$. The BSE, so derived, has as its kernel $(K)$ precisely the structure of the 4-fermion interaction assumed for the input Lagrangian, thus providing a logical (fleld-theoratic) basis for the BS kernel (K) Which now intended to serve for an effective description of the (non-perturbative) 
gluon propagator. The second aspect of the BSE so constructed is that the quark propagators involved in this equation are non-perturbative $S_{F}^{\prime}$-functions whose dynamical mass-functions $m\left(p^{2}\right)$ have been brought about in the first place through the CSB solution of the corresponding SDE, even though they did not appear in the input Lagrangian (with current quarks).

The basic idea behind this philosophy is hardly new, having been addressed by several people in the last decade $11-15)$, working mostly in the QCD coulomb gauge. The general result is that the chirally symmetric vacuum becomes unstable for the strong coupling constant $\alpha_{s}$ exceeding a critical value $\alpha_{c}(\gtrsim 1)$. However the key mechanism for CSB, viz., mass generation through a non-perturbative solution of the SDE-- The Gap Equation-- seems to be valid more generally for other phenomenological constructions with vector-exchange-type confining interactions, linear ${ }^{13)}$ or harmonic ${ }^{14)}$, as well. The same formalisms ${ }^{13-15)}$ also suggest that in the chiral Iimit $\left(m_{c}=0\right)$ the content of the gap equation is equivalent to the bound state BSE (with full propagators) corresponding to a zero mass pseudoscalar (pion), so that the masslessness of the pion is a rather general consequence of the dynamical mass generation for the quark. Further, the mass-function $m\left(p^{2}\right)$ of the quark can be identified with the vertex-function $\Gamma_{5}(q, P)$ of the pseudoscalar bound state in its zero mass limit.

Turning the problem the other way around, it wuold thus appear that an input vector-type confining kernel for a Bethe-Salpeter equation ${ }^{16)}$ possessing a natural property of chiral invariance, offers interesting possibilities for a realistic simulation of the NJL-mechanism, wherein the role of the cut-off parameter $(\Lambda)$ is now played by some natural mass-scale characterising the confining interaction itself. Such an interpretation should go a long way towards extending the scope of the NJL picture ${ }^{2}$ by allowing it to link up the mass-spectral predictions ${ }^{16)}$ with those of hadronic transition amplitudes ${ }^{17)}$ via standard quark loops. Though phenomenologically motivated, such a point of view should nevertheless help in bridging some rather big numerical gaps between the predictions of the classical NJL-model and other QCD-motivated effective Lagrangian descriptions ${ }^{18)}$, as noticed in the recent literature ${ }^{19)}$. Attempts on these lines are again not new ${ }^{14,9)}$, were it not for the rather inadequate attention to the phenomenological choice of the input 4-fermion interaction 14,9 ).

Perhaps a more serious concern towards a realistic construction of the BS kernel (vector-type) was evidenced in the effort of this group which was started quite early ${ }^{16-17)}$ with a conscious attention paid to its two-way predictabilityspectra on the one hand ${ }^{16)}$ and transition amplitudes on the other ${ }^{17)}$. The original motivation for the vector-type choice was not so much its chiral symetry property (which nevertheless existed!) as a conscious concern for a common confining mechanism 
for both qq̄ and qqq hadrons within a unified framework ${ }^{16)}$. Through several subsequent refinements on the initial formulation ${ }^{16-17)}$ in which the requirement of Lorentz-Covariance of the confining interaction had been the guiding consideration $^{20)}$, the model 21,6 ) with three basic constants and the input quark masses was able to account for the spectra of mesons $\left(Q \bar{Q}^{21}\right), Q \bar{q}$ and $q \bar{q}^{22}$ ) as well as of baryons, non-strange ${ }^{23)}$ and strange ${ }^{24)}$, within the same parametric structure. It was also able to provide a good description of several transition amplitudes 25 ), albeit in terms of the null-plane-ansatz(NPA) for the vertex function $\Gamma_{H}(q, P)$, which constrained it 22 lie on specified $3 D$ hypersurfaces $\left(q \cdot P=0, p_{i}{ }^{2}+m_{i}{ }^{2}=0\right.$, etc.) ${ }^{6}$. However this restriction has since been overcome through a natural Lorentz-covariant generalization $^{26}$ ) of the instant approximation--termed the Covariant-Instantaneity-Ansatz 26) -- which fully preserves ${ }^{26,27)}$ the spectral predictions $21-24$ ) of the null-plane formalism 20,6$)$ while giving formally unrestricted validity to the vertex function $\Gamma_{H}(q, P)$ over the entire $4 D$ space 26,27$)$.

In view of these distinct observational successes of this (two-tier) form $^{6}$ for of dynamics, a proper perspective of physics demands a closer look at the fuller chiral implications of the underlying 4-fermion Lagrangian,with a view to 1 inking up its analytic (and parametric) structure more explicitly with the NJL picture. This should hopefully provide, well beyond the specific model on hand 6,26$)$, some answer to more basic questions such as how far it is meaningful to relate the hadronic spectroscopy sector to the less trivial properties of the vacuum involving quark loops with non-trivial propagators, employing the (micro-causal) vehicle of the hadron-quark vertex function. A minimal condition for such extrapolation is of course a fully Lorentz-Covariant structure for the 4-fermion interaction (the BS kernel), so as to give a formal meaning to its crossing properties, provided the other analytic conditions (e.g., convergence of the loop integrals without arbitrary cut-offs) are satisfied. Since, as will be shown below, our Covariant model 26-27) does satisfy these requirements, we have specifically investigated and found the following results:

A) Self-consistent determination of $\mathrm{m}_{\mathrm{NJL}}(=300 \mathrm{Mev})$ from the Gap equation to be compared with the input value $m_{q}=265-270 \mathrm{Mev}$ employed for spectroscopy ${ }^{21-24)}$. B) An explicit relation like ${ }^{2}{ }_{\pi}^{\alpha} \mathrm{m}_{c}$, consistently with the chiral limit, as well as a separate determination of the sigma mass ( $\left.M_{\sigma}=857 \mathrm{Mev}\right)$. C) Direct evaluation of $\langle\bar{q} q\rangle_{0}$ as a convergent integral over the mass function m( $p^{2}$ ) as determined $\operatorname{from} \Gamma_{\pi}(q, P)$ of the BS mode1 26-27) in the chiral 1imit, leading to the parameterfree value of $(219 \mathrm{Mev})^{3}$.

Two independent estimates of $m_{c}$ from B) and $C$ ) are 21.8 and 17.8 (Mev) respectively. 
The Gap Equation (SDE)

Before discussing these results we shall briefly sketch their respective derivations, omitting most inessential details. To that end we start with the two basic equations, SDE for $\Sigma(p)$ and $B S E$ for $\Gamma_{H}(q, P)$. We shall use the momentum relations $p_{1,2}=\frac{1}{2} P \pm q, p_{1,2}^{\prime}=\frac{1}{2} P \pm q^{\prime}$ between the quark momenta $p_{i}, p_{i}^{\prime},(1=1,2)$, hadron momentum $P$ and the relative momenta $q, q^{\prime}$. In this notation the exchanged momentum (gluon-like) is $\mathrm{k}=\mathrm{q}-\mathrm{q}^{\prime}$; all these are 4 -vectors.

SDE: $\Sigma(p)=\frac{4}{3} i \int \frac{d^{4} k}{(2 \pi)^{4}} D_{\mu \nu}(k) \gamma_{\mu-} s_{F}^{\prime}\left(p^{\prime}\right) \gamma_{\nu}$

$S_{F}^{\prime}$ is the full propagator related to the mass operator $\Sigma(p)$ by

$$
\Sigma(p)+i \gamma \cdot p=S_{F}^{\prime-1}(p)=A\left(p^{2}\right)\left[i \gamma \cdot p+m\left(p^{2}\right)\right] \text {, }
$$

thus defining the mass function in the chiral limit $\mathrm{m}_{\mathrm{c}}=0$.

BSE:

$\Gamma_{H}(q, P)=-\frac{4}{3} i \int \frac{d^{4} q^{\prime}}{(2 \pi)^{4}} D_{\mu \nu}\left(q-q^{\prime}\right) \gamma_{\mu} s_{F}^{\prime}\left(\frac{1}{2} P+q^{\prime}\right) \Gamma_{H}\left(q^{\prime}, P\right) s_{F}^{\prime}\left(-\frac{1}{2} P+q^{\prime}\right) \gamma_{\nu}$

In each equation, the color factors have been replaced by Casimirs, and the propagator $D_{\mu \nu}(k)$ in the Landau gauge has the form $\left(\delta_{\mu \nu}-k_{\mu} k_{\nu} k^{-2}\right) \dot{D}(k)$ where $D(k)$ incorporates the effect of the non-perturbative 4-fermion interaction. In the BS model in which we are going to describe the results, $D(k)$ has the form 6,26 :

$$
D(k)=D(\hat{k})=\frac{3}{4}(2 \pi)^{3} \omega_{0}^{2} \cdot 2 m_{q} \alpha_{s}\left(4 m_{q}^{2}\right)\left[\nabla_{\hat{k}}^{2}+c_{0} \omega_{0}^{-2}\right] \delta^{3}(\hat{k})
$$

At this stage a few words are in order regarding the analytic structure of $D(k)$ in $k$ and $m_{q}$ ( the constituent mass ). First, its dependence on $\hat{k}_{\mu}=k_{\mu}-k \cdot P_{\mu} P^{-2}$, the momentum transverse to $P_{\mu}$, is the chief ansatz of our model; see ref(26-27)for details. The quantity $\hat{k}_{\mu}$ which is thus $3 D$ in content $(\hat{k} \cdot P \equiv 0)$, has the important property $\hat{\mathrm{k}}^{2}>_{0}$ all over $4 \mathrm{D}$ space, and also possesses a well-defined limit as $\mathrm{P}_{\mu} \rightarrow 0$, since it depends only on the direction $\hat{n}_{\mu}=P_{\mu} / \sqrt{P^{2}}$ of $P_{\mu}$ and notpn its magnitude. As a result, the integrand on the right hand side of the gap equation (1) technically "sees" this direction (signalling the CSB effect?) but it will not show up in the result for the integral. On the other hand, the explicit dependence of $D(k), E q .(4)$, on the constituent mass $m_{q}$ is, within our model, an integral part of the dynamics of the gap equation, since for $\mathrm{m}_{c} \rightarrow 0, \mathrm{~m}_{\mathrm{q}} \rightarrow \mathrm{m}_{\mathrm{gL}}$, the quantity which is supposed to represent the self-consistent solution for $m\left(p^{2}\right)$ corresponding to $p^{2}=-m_{N}^{2}$. This in our view constitutes a rather severe test of the parametric structure of the model itself. Finally the quantities $c_{0}(=0.27)$ and $\omega_{0}(=158 \mathrm{Mev})$ are constants of the model which control the spectra $21-24)$.

We first solve the gap equation, borrowing a well-known result from the literature that in the Landau gauge for $\mathrm{D}_{\mu y}$ (that we have employed), the function $A\left(p^{2}\right)$ does not undergo remormalization and can be set equal to unity ${ }^{28)}$. Using this result in $\mathrm{Eq}(2)$ and introducing the full structure (4) for $D(k)$, the gap equation (1) reduces straightforwardly to 
$m\left(p^{2}\right)=\frac{3 i}{\pi} \int d^{3} \hat{k} d k_{10}\left(m_{q} \cdot \alpha_{s}\right)\left[\omega_{0}^{2} \nabla_{\hat{k}}^{2}+c_{0}\right] \delta^{3}(\hat{k}) \frac{m^{\left(p^{\prime 2}\right)}}{p^{\prime 2}+m^{2}\left(p^{\prime 2}\right)}$

where $p^{\prime}=p-k$, and $k_{1 \mu}=k \cdot p p_{\mu} / p^{2}$ is the longitudinal component of $k_{\mu}$. Because of the $\delta^{3}(\hat{k})$ function, tha integral is essentially over $\mathrm{dk}_{10}=\mathrm{dp}^{\prime}{ }^{\prime}$ io and gives the pole value $\mathrm{p}^{\prime}{ }_{10}=\mathrm{m}\left(\mathrm{p}^{\prime}{ }_{10}\right)=\mathrm{m}_{\mathrm{NJL}}$, since the variation w.r.t. $\hat{\mathrm{p}}_{\mu}^{\prime}=-\hat{\mathrm{k}}_{\mu}$ is now irrelevant. Evaluation of the integral leads simply to

$$
\mathrm{m}_{\mathrm{NJL}}=3\left(\mathrm{~m}_{\mathrm{q}} \alpha_{\mathrm{s}}\right) \mathrm{m}_{\mathrm{NJL}}{ }^{-2}\left(3 \omega_{0}^{2}-\mathrm{c}_{0} \mathrm{~m}_{\mathrm{NJL}}^{2}\right) \text {. }
$$

The identification $m_{q} \rightarrow m_{N J L}$ in the factor $\left(m_{q} \alpha_{s}\right)$, where ${ }^{22)}$

$$
\alpha_{s}=(6 \pi / 29) / \ln \left(10 . m_{q}\right) \text {, in Gev units, }
$$

yields an independent, self-consistent, estimate $\mathrm{m}_{\mathrm{NJ}}=300 \mathrm{Mev}$. This may be compared with the empirical value $m_{q}=265-270 \mathrm{Mev}$ employed to fit the spectra ${ }^{21-24)}$.

Pion and sigma masses away from the chiral limit:

Our next task is to calculate the physical mass of the pion away from the chiral limit $\left(m_{c} \neq 0\right)$. To that end it is first necessary to check the complete equivalence of the BS equation (3), with $\Gamma_{H}=\underline{X}_{5} \Gamma_{\pi}(q)$ for the pion, and the gap equation (1) in the limit $P_{\mu} \rightarrow 0$. This condition is easily seen to be satisfied, in conformity with other investigaticns, provided the vertex function $\Gamma_{\pi}(q, P)$ is identified with the mass function $m\left(p^{2}\right)$ in the chiral $\left(p_{\mu} \rightarrow 0\right)$ 11mit. Both equations of course reduce to (5) in the present case. To move away from the chiral limit, we now concentrate on the $B S E$, Eq(3), with $P^{2}=-M^{2}$, and simultaneously introduce the current mass $m_{c}$,closely following the argument of ref.(29), via the replacement

$$
m\left(p_{i}^{2}\right) \rightarrow m_{N J L}+m_{c} \equiv m_{q} \text { (constituent), }
$$

ignoring the small variations in $m\left(p^{2}\right)$ slightly away from its chiral limit pole value $m=m_{N J L}$; see argiment after Eq. (5). Eq. (3) now reads(after eliminating the Dirac matrices) as

$$
\Gamma_{\pi}(q, P)=\frac{3 i}{\pi}\left(m_{q} \cdot \alpha_{s}\right) \int d^{3} \hat{q}^{\prime} d q_{10}^{\prime} \Gamma_{\pi}\left(q^{\prime}, p\right)\left[\omega_{0}^{2} \nabla_{\hat{i}^{\prime}}^{2}+c_{0}\right] \delta^{3}\left(\hat{q}^{\prime}-\hat{q}\right) f\left(\hat{q}^{\prime 2}, q_{1}^{\prime 2}\right.
$$

where $q_{1}^{\prime}=1 q_{10}^{1}, m_{q}$ now stands for the constituent mass, Eq. (8), and.

$f(x, y)=\left(m_{q}^{2}+x+y+m^{2} / 4\right) /\left[\left(m_{q}^{2}-i \varepsilon+x+y-M^{2} / 4\right)^{2}+M^{2} y\right]$

An approximate solution of (9) now follows on lines closely analogous to Eq. (5), with the further observation that since the vertex function $\Gamma_{\pi}(q, P)$ equals the mass function $m(q)$ in the chiral limit, variations in this quantity on both sides of Eq. (9) can be ignored if we work in the neighbourhood of the point $\hat{q}_{\mu}=0$ (which implies that $\hat{q}_{\mu}^{\prime}=0$ inside the integral). This leads, on $d^{3} \hat{q}^{\prime}$ integration, to

$$
1=\left.\frac{31}{\pi}\left(m_{q} \cdot \alpha_{s}\right) \int d q_{10}^{\prime}\left[c_{0}+6 \omega_{0}^{2} \frac{\partial}{\partial \hat{q}^{2}}\right] f\left(\hat{q}^{2}, q_{\perp}^{\prime 2}\right)\right|_{\hat{q}^{2}=0}
$$


The subsequent integration is easily performed through a Wick rotation and leads in an exact fashion to

$$
1=3 \alpha_{s}\left\{\frac{3 \omega_{o}^{2}\left(m_{q}{ }^{2}+M^{2} / 4\right)}{\left(m_{q}^{2}-\frac{1}{4} M^{2}\right)^{2}}-\frac{c_{0} m_{q}{ }^{2}}{\left(m_{q}{ }^{2}-\frac{1}{4} M^{2}\right)}\right\}
$$

which is fully compatible with the gap equation (6) when rewritten (for $m_{c}=0$ ) as

$$
1=3 \alpha_{s}\left(3 \omega_{0}^{2} \mathrm{~m}_{\mathrm{NJL}}{ }^{-2}-\mathrm{c}_{0}\right)
$$

Subtracting (13) from (12) and ignoring the small difference between $m_{q}$ and $m_{N J L}$ inside $\alpha_{s}$, gives a relation between $M_{\pi}^{2}$ (.M equals $M_{\pi}$ in this case) and $m_{c}$ (see Eq. (8)), which in the first order in $m_{c}$ reads:

$$
8 \mathrm{~m}_{\mathrm{N} J} \cdot \mathrm{m}_{\mathrm{c}}=3 \mathrm{M}_{\pi}^{2}\left(1-\frac{1}{9} \mathrm{C}_{0} \mathrm{~m}_{\mathrm{NJ}}{ }^{2} \omega_{0}^{-2}\right)
$$

and yields the estimate $m_{c}=21.8 \mathrm{Mev}$. This number is admittedly high, but a

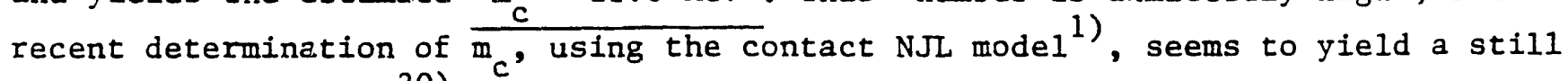
higher value $\left.(34 \mathrm{Mev})^{30}\right)$. On the other hand, a somewhat lower value $(17.8$ Mev ) is obtained from the GMOR relation ${ }^{31)}$, using the value of $\langle q \bar{q}\rangle_{0}$ determined directly within this model (see further below).

Calculation of the sigma-mass proceeds on very similar lines wherein the vertex function $\Gamma_{\mathrm{H}}$ has the dominant structure ${ }^{25,6)} \Gamma_{\mathrm{H}}=1 \gamma \cdot \hat{q} \Gamma_{\sigma}(\mathrm{q}, \mathrm{P})$. After substitution in Eq.(3), and tracing out the Dirac matrices, the equation for $\Gamma_{\sigma}$ reads

$$
\begin{gathered}
\Gamma_{\sigma}(q, P)=\frac{3 i}{4} \pi\left(m_{q} \cdot \alpha_{s}\right) \int d^{3} \hat{q}^{\prime} d q_{10}^{\prime} \Gamma_{\sigma}\left(q^{\prime}, P\right)\left[\omega_{0}^{2} \nabla_{\hat{q}^{\prime}}{ }^{2}+c_{0}\right] \delta^{3}\left(\hat{q}-\hat{q}^{\prime}\right) . \\
\otimes \frac{2 \hat{q} \cdot \hat{q}^{\prime}}{\hat{o}^{2}} \cdot f\left(\hat{q}^{\prime 2}, q_{1}^{\prime 2}\right),
\end{gathered}
$$

where the f-function continues to be given by (10). The reduced vertex function $\Gamma_{\sigma}$ no longer enjoys the chiral property of $\Gamma_{\pi}(q, P)$, but it is still possible to work in the neighbourhood of $\hat{q}_{\mu}=0$ just as in the pion case, thanks to the presence of $\delta^{3}\left(\hat{q}-\hat{q}^{\prime}\right)$ on the right hand side of (15). Further, since the integral is again dominated by the pole value of $q_{10}^{\prime}$, determined by the denominator of the same function $f(x, y)$ as in the pion case, it is again possible in the first approximation to ignore the variations of $\Gamma_{\sigma}$ w.r.t. $q_{10}^{\prime}$ in the neighbourhood of this pole. Eq. (15) then reduces after $d^{3} \hat{q}^{\prime}$ integration to

$$
1=\frac{3 i}{\pi}\left(m_{q} \cdot \alpha_{s}\right) \int d q_{10}^{\prime}\left[\frac{1}{2} c_{0}+5 \omega_{0}^{2} \frac{\partial}{\partial \widehat{q}^{2}}\right] f\left(\hat{q}^{2}, q_{10}^{\prime 2}\right) \mid \hat{q}^{2}=0
$$

as the counterpart of (11). The integration over $\mathrm{dq}_{10}^{\prime}$ can again be carried out in an exact fashion, giving rise to the exact result

$$
1=3 \alpha_{s}\left[-\frac{1}{2} C_{0} z+15 \omega_{0}^{2}\left(z+2 z^{2}\right) m_{q}^{-2}\right] ; \quad z=m_{q}{ }^{2} /\left(m_{q}{ }^{2}-M^{2} / 4\right) \text {. }
$$

While it is no longer important to keep the distinction between $m_{q}$ and $m_{N J L}$ in the 
sigma case, the exact evaluation of the integral has been an important step in

view of the(expected)proximity of $M=M_{\sigma}$ to the value of $2 m_{q}$. Using the gap equation to eliminate $\alpha_{S}$, gives the explicit formula $\left(\mathrm{M}^{2} / 4 \mathrm{~m}_{\mathrm{q}}^{2}\right)=1+\left[(15 / 2 \lambda)^{2}+(30 / \lambda)\right]^{1 / 2}-(15 / 2 \lambda) ; \lambda=3-\mathrm{c}_{0} \mathrm{~m}_{\mathrm{q}}{ }^{2} \omega_{0}^{-2}$ yielding the value $M_{\sigma}=857 \mathrm{Mev}$. Our estimate is significantly higher than the canonical NJL value $\frac{\sigma}{2 \mathrm{~m} J}(=600 \mathrm{Mev})$, and may be attributed to the non-locality of the interaction. Our 'chiral' determination is nevertheless consistent with the more conventional value of the mass of this $L=1$ state via the $3 D$ form of the BSE 21-22), analogously to potential models ${ }^{5,6)}$ which tend to give much higher values than $2 \mathrm{~m}_{q^{\circ}}$.

The quark condensate in vacuum- $\langle\mathrm{q} \bar{q}\rangle_{0}:$

Finaily we offer a non-trivial prediction of the model, viz., the value of $\langle q \bar{q}\rangle_{0}$, directly in terms of the mass function $m\left(p^{2}\right)$ :

$$
\langle q \bar{q}\rangle_{0}=N_{c} N_{f} \cdot \frac{-i}{(2 \pi)^{4}} \int d^{4} p \operatorname{Tr} s_{F}^{\prime}(p)=\frac{-24 i}{(2 \pi)} 4 \int d^{4} p \frac{m\left(p^{2}\right)}{m^{2}\left(p^{2}\right)+p^{2}}
$$

Unlike the two previous cases where a full determination of the momentum dependence of the mass function was not necessary, thanks to the multiplicative presence of the gluon propagator (4), we now need an explicit formula for $m\left(p^{2}\right)$. The key to this determination lies in the observation that in the chiral limit. $P=p_{1}+p_{2}=0$, the pion vertex function $\Gamma_{\pi}(q, P)$ reduces to $m\left(p^{2}\right)$, where $p_{\mu}$ is the 4-momentum of either constituent. Now $\Gamma_{\pi}(q, P)$ is fully determined in the BS model in terms of the transverse momentum $\hat{q}_{\mu}$, viz., 26-27)

$$
\begin{aligned}
& \Gamma_{\pi}(q, P)=N_{B S} D(\hat{q}) \otimes \phi(\hat{q}) ; \phi(\hat{q})=\exp \left(-\frac{1}{2} \hat{q}^{2} / \beta^{2}\right) \\
& D(\hat{q})=4 \omega_{\hat{q}}\left(\omega_{\hat{q}}{ }^{2}-M^{2} / 4\right) ; \omega_{\hat{q}}{ }^{2}=m_{q}{ }^{2}+\hat{q}^{2} .
\end{aligned}
$$

And since in the chiral limit we also have $\hat{q}^{2}=\hat{p}^{2}$, the mass function in our Lorentzcovariant BS model finally works out as

$$
m\left(p^{2}\right)=m\left(\hat{p}^{2}\right)=m_{q}^{-2} \omega \hat{\beta}^{3} \exp \left(-\frac{1}{2} \hat{p}^{2} / \beta^{2}\right)
$$

where the normalization has been fixed by the condition $m(0)=\mathrm{m}_{q}=m_{N J}$; see (8). Eq. (22) is the central ingredient for the determination of $\langle q \bar{q}\rangle_{0}$ from Eq. (19). In this case the integration over the longitudinal variable dp 10 is carried out first to yield

$$
\pi^{2}\langle q \vec{q}\rangle_{0}=6 \int_{0}^{\infty} \hat{p}^{2} d \hat{p} m\left(\hat{p}^{2}\right)\left[\hat{p}^{2}+m^{2}\left(\hat{p}^{2}\right)\right]^{-1 / 2}
$$

which gives a perfectly convergent integral in terms of the function (22). A simple quadrature, using the BS model value

$$
\langle\mathrm{q} \overline{\mathrm{q}}\rangle_{0}=(219 \mathrm{Mev})^{3}
$$$$
\text { 22) } \beta^{2}=0.031 \mathrm{Gev}^{2}, \text { gives }
$$

This quantity can be employed in conjunction with the GMOR relation 31 ) $M_{\pi}^{2} f_{\pi 3}^{2}=m_{c}\langle q \bar{q}\rangle_{0}$ to find an independent estimate for $m_{c}$ since both $M_{\pi}$ and $f_{\pi 3}$ 
have been pre-determined in the model 22,27$)$ :

$$
M_{\pi}=140 \mathrm{Mev}\left(\text { ref.22); } \mathrm{f}_{\pi 3}=138 \mathrm{Mev} / \sqrt{2} \quad\right. \text { (ref.27). }
$$

A direct substitution yields the value $m_{c}=17.8 \mathrm{Mev}$, which is somewhat lower than

the other estimate $\mathrm{m}_{\mathrm{c}}=21.8 \mathrm{Mev}$, Eq. $(14 \overline{\text {, based }} \overline{\text { on the }}$ consistency with the gap equation.

Resume and discussion

We have endeavoured to draw attertion to the practical possibilities of a realistic simulation of the NJL mechanism of dynamical mass-generation through a close interplay of the Schwinger-Dyson and Bethe-Salpeter equations both of which are derivable ${ }^{8)}$ from a Lagrangian with a given 4 -fermion coupling of a sufficiently general nature (not necessarily local). The only non-trivial requirement for such effective Lagrangian is the property of chiral invariance, one which is met rather naturally from a vector-exchange type structure without the restriction to a contact form envisaged in the original model ${ }^{1}$. Such a relaxation has the advantage of keeping a conscious link with the vital sector of spectroscopy through some natural mass scale characteristic of a confining interaction, a property which the cut-off parameter $(\Lambda)$ of the contact interaction does not possess. Though the idea of interplay of SDE and BSE with an extended vector-type 4-fermion Lagrangian in not new $^{8-15)}$, the cruciai aspect we have tried to emphasize is the possibility of a fuller exploitation of the idea in a practical enough manner which helps retain the spectroscopic link as an integral part of the physics. In this respect the BS program we have been pursuing during the last several years ${ }^{20-27)}$ seems to offer a natural testing ground for the chiral mechanism of NJL because of its built-in vector-type structure on the one hand, and its prior spectroscopic check on a fairly extensive scale $\mathrm{e}^{21-25)}$ on the other. The underlying chiral aspects of the formalism which had so far remained untapped, have now been studied in this paper, being facilitated by a recent refinement ${ }^{26-27)}$ of the formalism which now gives it a manifestly Lorencz- covariant structure as well as a firmer theoretical basis to its 'two-tier' philosophy. Indeed, the exact interconnection between the $3 D$ and $4 D$ forms of the BSE brought about by the Covariant Instantareity Ansatz(CIA) 26 ) (which gives a 3D support to the BS kernel) has proved a major factor in providing the Lorentz- covariant structure, $\Gamma(\hat{q})=D(\hat{q}) \otimes \phi(\hat{q})$ for the vertex function without which this necessarily 4D investigation would not have been possible.

As a first check, our self-consistent estimate of $m_{N J}(=300 \mathrm{Mev})$ from the gap equation is not too far from the input value of $\mathrm{m}_{q}(=265-270 \mathrm{Mev})$ employed for the spectroscopy ${ }^{21-24)}$. As a(belated) check on our spectroscopy, through this chiral interplay of the SDE and the BSE we also seem to have a clearer understanding(by hindsight) as to why the pion mass had come out 'rathar well' through the more orthodox (3D) form of application of the $\mathrm{BSE}^{21-22)}$, a result which had then remained unexplained ${ }^{21)}$ : It is the chiral property of the vector-type interaction which has 
been playing the central role, perhaps covertly -Within the simple-minded $3 \mathrm{D}$ BSE 14,21 ) but now directly manifesting through the proportionality of $M^{2} \pi$ to the current mass $\mathrm{m}_{c}$, Eq. (14). Nevertheless this direct(chiral)check on the input parameters has helped provide a useful calibration to the more conventional form of spectroscopy. As a related item, the significantly larger sigma mass ( $857 \mathrm{Mev}$ ) than $2 \mathrm{~m}_{\mathrm{NJL}}$ is probably due to the extended nature of the interaction. This is only a technical violation of the NJL mode1 ${ }^{2}$ ) but more in conformity with the normal spectroscopy of an $L=1$ state.

A more serious test of the non-perturbative nature of the interplay of the BSE versus SDE has been provided by the $\langle q \bar{q}\rangle_{0}$ calculation which has required a non-trivial form of the mass function ( for a properly convergent integral) via its chiral connection with the pion vertex function in the $P_{\mu}=0$ 1imit. This vital component has now been provided independently by the Bethe-Salpeter sector of the dynamics $^{26-27)}$ in the $4 D$ form $\Gamma(\hat{q})=D(\hat{q}) \otimes \phi(\hat{q})$. Therefore the value $(219 \mathrm{Mev})^{3}$ for the condensate carries a strong signature of the spectroscopy through the Lorentz-covariant chiral vehicle of the vertex function, with no arbitrary cutoff parameter needed for its determination. The two independent determinations of $m_{c}$ from/different premises ( gap equation vs. GMOR ${ }^{31}$ ) relation), with a rather small spread ( $21.8 \mathrm{Mev}$ vs. $17.8 \mathrm{Mev}$ ) constitute a welcome check on the self-consistency of the internal dynamics, one which is symbolised by the above $4 \mathrm{D}$ form of $\Gamma(q)$.

To conclude, it is perhaps in order to make some remarks on the significance of the Covariant-Instantaneity-Ansatz 26-27) that has played a key role in linking spectroscopy with a Lorentz-covariant vertex function. From a physical poir.t of view the nearest it comes is to the spirit of the coulomb gauge ${ }^{8-14)}$ investigations of the chiral confinement problem, by and large within the instantaneous approximation. This celebrated approximation which has had a long history, has been defended afresh on more general grounds ${ }^{32)}$. Therefore the CIA aspect of our formulation has ramifications of a more general nature than can be attributed to the specific model alone ${ }^{26-27}$ : and would seem to give a meaning to attempts to link spectroscopy with the wider NJL picture of confinement through CSB.

This work was completed (and written) during the visit of one of us (ANM) to the INT in August 1991 under Program V, and the underlying ideas found an exposure through two seminars on the subject, at the Univ.of Maryland and the Institute for Nuclear Theory respectively. ANM is grateful to Prof. Henley for hospitality, to the organisers ( Prof G.Miller and Prof G.Karl) for invitation to Program V; and to DOE for financial support.

\section{References}

1. Y.Nambu and G.Jona-Lasino, Phys,Rev, 122, 345 (1961)

2. Y,Nambu in V.Telegdi Festschrift, Ed K,Winter, NH Pub Co, Amsterdam, 1988

3. H.D.Politzer, Nucl.Phys, B117, 397 (1976)

4. e.g.,D.Kahana and U.Vog1, Phys.Letters 244B, 10 (1990) 
5. E.Eichten et a1, Phys.Rev.Lett. 34,369 (1975)

6. See for a review S.Chakrabarty et al, Prog.Part. Nucl. Phys.22, 43-180 (1989)

7. V.Pervushin,D.Ebert,H.Reinhardt, Sov.J.Part.Nuc1.10,1114 (1979)

8. H.J.Munczek, Phys.Rev.D25, 1579 (1982)

9. Yu.L.Kalinovsky et al, Phys.Letters 231B, 288 (1989)

10. R.T.Cahill et a1, Phys.Rev. D32, 2422 (1985)

11. V.A.Miransky et al, Phys. Letters 100B, 157(1981)

12. J.Finger and J.Mandula, Nucl.Phys. B199, 168(1982)

13. S,Adler and A.C.Davis, Nucl.Phys.B244, 469 (1984)

14. A.Le Yaouanc et al, Phys. Rev.D29, 1233 (1984)

15. R.Delbourgo and M.D.Scadron, J.Phys G5, 1621 (1979)

16. A.N.Mitra and I.Santhanam, Z.f.Phys. C8, 25, 33 (1981)

17. A.N.Mitra,D.Kulshreshtha, Phys.Rev.D26,3123(1982); D28, 588 (1983)

18. J.Gasser, H.Leutwyler, Nucl.Phys. B250, 465, 539 (1985)

19. e.g., T.H.Hanson et al, Nuc1. Phys.B335, 67 (1990)

20. N.N.Singh et al, Few-Body Systems 1,47 (1986)

21. A.Mittal and A.N.M itra, Phys.Rev.Lett. $\underline{\text { 57}}$ : 290 (1986)

22. K.K.Gupta et al, Phys.Rev.D42, 1604 (1990)

23. D.S.Kulshreshtha and A.N.Mitra, Phys.Rev.D37, 1268 (1988)

24. J.T.Londergan and A.N.Mitra, Int1.J.Mod.Phys.ㅌ, 2659 (1991)

25. N.N.Singh and A.N.Mitra, Phys. Rev. D38, 1454 (1988)

26. A.N.Mitra and S.Bhatnagar, Intl. J. Mod. Phys.A7 (1991); in press

27. S.Bhatnagar, A.N.Mitra, D.S, Kulshreshtha, Phys.Letters 263B, 485 (1991)

28. D.Atkinson and P.W.Johnson, Phys.Rev.D41, 1661 (1990)

29. R.T.Cahill et al, Phys.Rev. D36, 2804 (1987)

30. T.Hakioglu and M.Scadron, Phys. Rev. D42, 941 (1990)

31. M.Gel1-Mann, R.Oaks, B.Renner, Phys. Rev. 175, 2 i95 (1968);

S.L.Glashow and S. Weinberg, Phys.Rev.Lett. $\frac{20}{2}, 224$ (1968)

32. e.g., N.S.Han and V.N.Pervushin, Fortschritte Phys. 37, 611 (1989) 

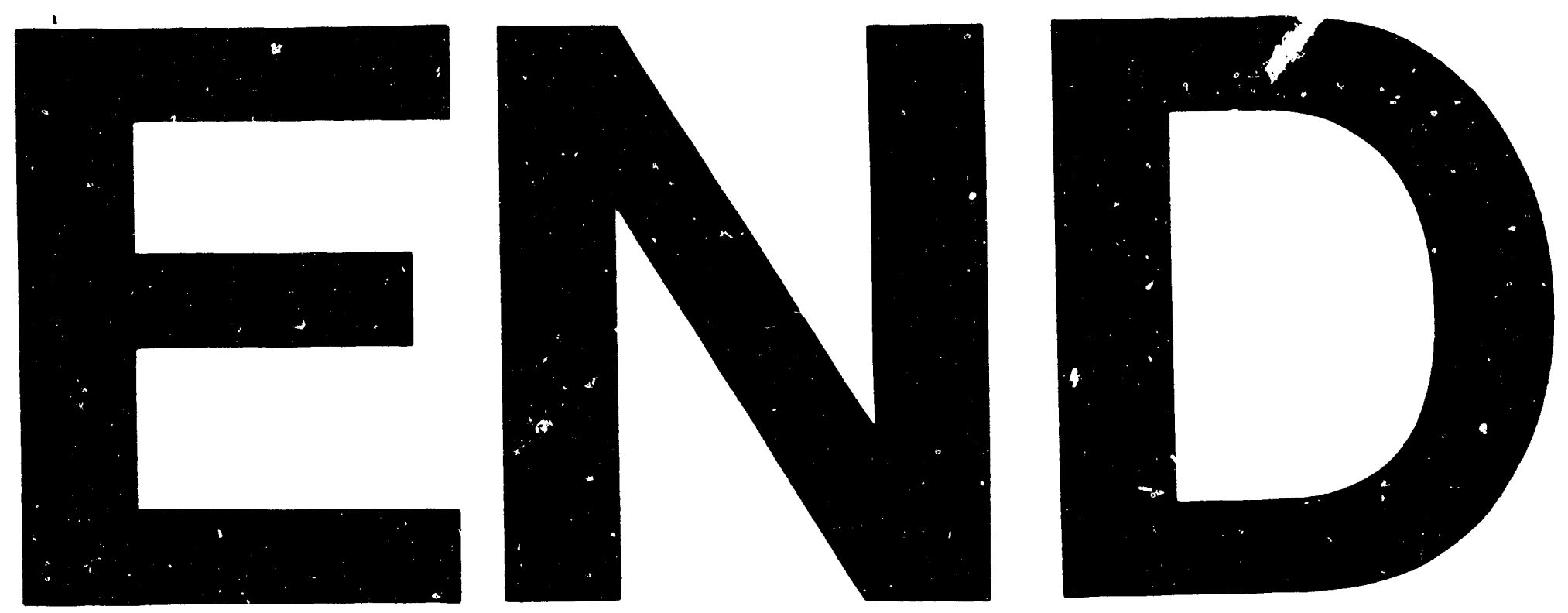

4
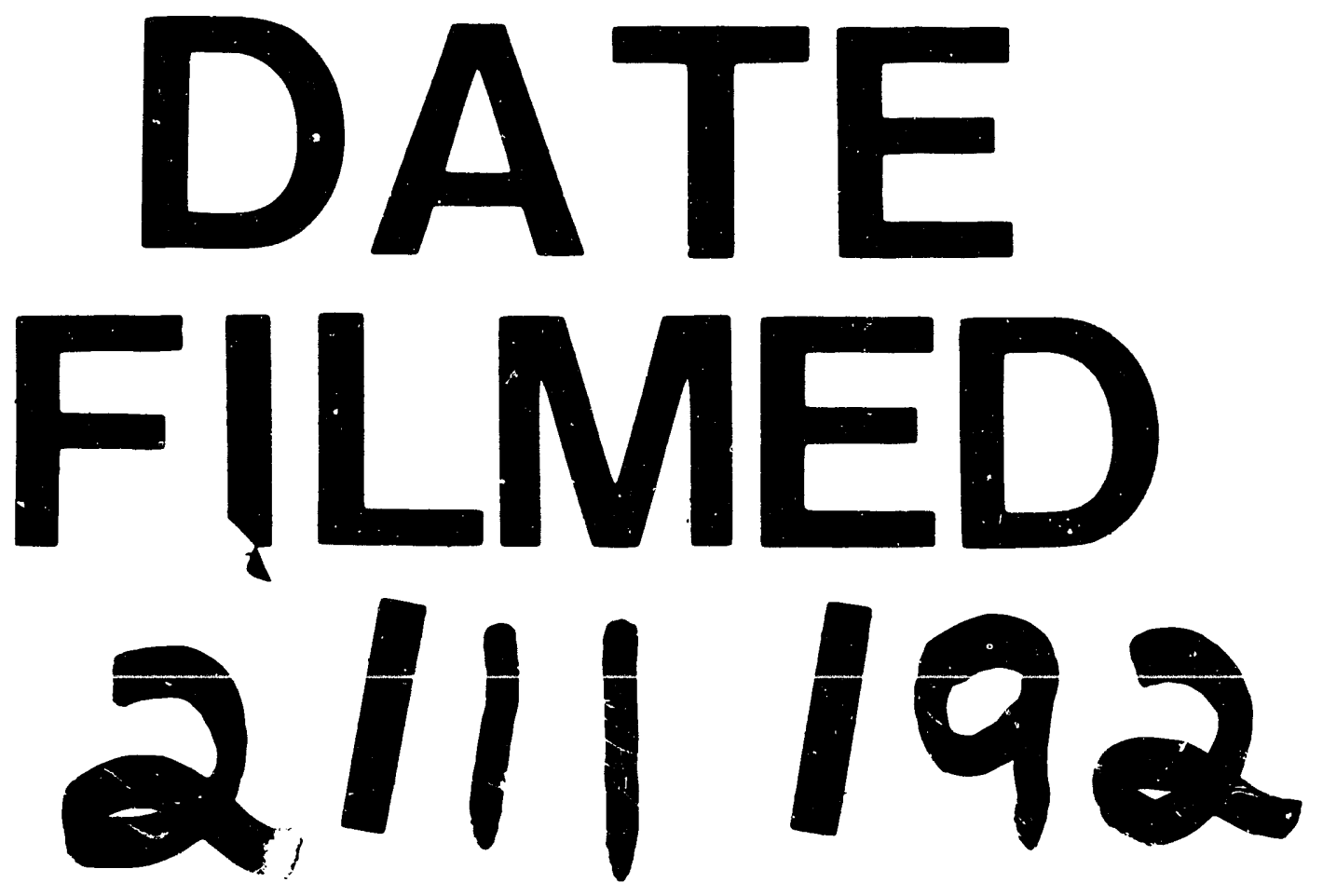

$I$ 
$=$ 